\title{
Análisis comparado de las políticas públicas de defensa de México y Brasil. Retos de una nueva contrainsurgencia*
}

\author{
Comparative analysis of public policies in \\ defense of Mexico and Brazil. Challenges of a \\ new counterinsurgency
}

\author{
Luís Alexander Montero Moncada** \\ Ángela Herrera*** \\ Liana Abril Pérez ${ }^{* * * *}$
}

Recibido: 29/01/2014

Aprobado: 14/03/2014

Disponible en línea: 01/07/2014

\section{Resumen}

La evolución de la doctrina de contrainsurgencia ha tenido en el escenario latinoamericano un espacio interesante para matizar las diferentes escuelas teóricas y para experimentar adecuaciones especiales según las necesidades imperantes en la región. En ese sentido, las políticas públicas de seguridad y defensa de México y Brasil ofrecen

\begin{abstract}
The evolution of counterinsurgency doctrine has had on the Latin American scene an interesting space to refine different theoretical schools and to experience special adaptations to the needs that prevail in the region. In this sense, public policies on security and defense of Mexico and Brazil offer patterns of challenges
\end{abstract}

doi:10.11144/Javeriana.PAPO19-2.acpp

* Articulo resultado de investigación asociado al proyecto titulado "Evolución de la doctrina de contrainsurgencia de 1947 a 2011". Escuela Militar de Cadetes. Dirección de Investigaciones. 2012. ${ }^{* *}$ Politólogo con énfasis en Relaciones Internacionales. Magíster en Análisis de Problemas Políticos, Económicos e Internacionales Contemporáneos. PhD. en Estudios Políticos y Relaciones Internacionales. Director de Investigaciones de la Escuela Militar de Cadetes. Profesor e Investigador experto en Seguridad, Defensa y Operaciones Especiales. Correo electrónico: Alexander.montero@gmail.com ${ }^{* * *}$ Profesional en Relaciones Internacionales y Estudios Políticos, Especialista en Ambiente y Desarrollo local. Actualmente se desempeña como investigadora de la Dirección de Investigaciones de la Escuela Militar de Cadetes General José María Córdova. Correo electrónico: anmaheca@gmail.com

**** Profesional en Relaciones Internacionales y Estudios Políticos, Especialista en Administración Financiera. Actualmente se desempeña como investigadora de la Dirección de Investigaciones de la Escuela Militar de Cadetes General José María Córdova.

Correo electrónico: investigacionasesor4@esmic.edu.co 
patrones claros de retos y amenazas que desarrollan nuevas formas de atacar a los Estados, así como las respuestas que se han concebido desde novedosas concepciones de contrainsurgencia.

\section{Palabras clave:}

contrainsurgencia; políticas de defensa; mafias; crimen organizado; fuerzas militares

\section{Cómo citar este artículo:}

Montero, L. A., Herrera, A. y Abril, L. (2014). Análisis comparado de las políticas públicas de defensa de México y Brasil. Retos de una nueva contrainsurgencia. Papel Político, 19 (2), 631-655. http://dx.doi.org/10.11144/ Javeriana.PAPO19-1.acpp and threats that develop new ways to attack the States, and the responses, from new concepts of counterinsurgency.

\section{Keywords:}

counterinsurgency; defense policy; gangs; organized crime; military forces 


\section{Introducción}

El presente documento tiene por objeto abordar el análisis de las políticas de defensa de los Estados brasileño y mexicano, desde una perspectiva comparada y en el marco de una serie de diferencias estructurales que alimentan la discusión con una interesante complejidad, la cual se explica, como se precisará en las líneas siguientes, en las realidades alternas de uno y otro país, mediadas tanto por nuevas lógicas de distribución del poder mundial, como por las reconocidas amenazas del siglo XXI dentro de las que resaltan el narcotráfico, el crimen transnacional y el crimen organizado; todo ello enmarcado en la herencia de regímenes con tradiciones de un profundo liderazgo o control militar sobre el poder político.

Para realizar este ejercicio comparado, abordaremos en primer lugar un planteamiento teórico en virtud del cual resulta posible describir y caracterizar las políticas de defensa como políticas públicas. En segundo y tercer lugar, realizaremos un análisis de la política pública de seguridad y defensa mexicana, tomando como puntos de referencia los documentos rectores de seguridad nacional mexicana y la reconfiguración de las relaciones cívico militares que ha caracterizado la estructura militar de Brasil con posterioridad al periodo de junta militar, respectivamente. En cuarto lugar, propondremos, con base en los acápites dos y tres, una serie de comparaciones entre una y otra política, apoyados en los elementos característicos de las políticas de seguridad y defensa democráticas, incluyendo la metodología de los libros blancos de la defensa, para así formular finalmente unas conclusiones y puntualizar algunos retos identificados en ambos análisis.

\section{Las políticas públicas como un proceso de gobierno. La necesidad de un direccionamiento hacia nuevas COIN}

La diferencia de lenguaje o fundamentalmente los retos propuestos por la escuela anglosajona, precursora de la discusión sobre políticas públicas, hacen que no sea unánime la definición misma del término de políticas públicas. La divergencia entre polity, politics y policy, hacen que cualquier intento de definición pueda encontrar tres, o al menos dos caminos posibles por recorrer, especialmente si se enfatiza en los conceptos de politics y policy. Por esta razón, se conciben nociones provenientes desde escuelas distintas pero que tienen en común un mismo concepto, que si bien tampoco permite universalidades, sí define o delimita la acción de las políticas públicas, así como el concepto de 'lo público’.

La noción de 'lo público', dicho sea de paso, aparece inmediatamente ligada al concepto de la seguridad, puesto que no hay servicio más público que el de garantizar la seguridad y la supervivencia a los individuos de un Estado. De hecho, la dimensión pública de la seguridad se erige en piedra fundamental de las teorías contractualistas del Estado desarrolladas por Hobbes y Rousseau, donde aquel -el Estado- nace precisamente para garantizar -antes de cualquier otra cosa- la seguridad a través de la renuncia de libertades de sus asociados o logrando el monopolio del ejercicio de la violencia. 
Así, encontramos análisis como los propuestos por Laswell (1992) quien plantea, tras analizar el impacto que tienen la guerra y las tensiones en materia de seguridad sobre la función de gobierno y la administración del poder, que se debe pensar fundamentalmente en la existencia de unas Ciencias de las Políticas a la hora de diseñar y promover las políticas públicas y su relación inmediata con la supervivencia misma del Estado. Laswell define entonces las Ciencias de las Políticas como:

[...] el conjunto de disciplinas que se ocupan de explicar los procesos de elaboración y ejecución de las políticas, y se encargan de localizar datos y elaborar interpretaciones relevantes para los problemas [que enfrenta el poder] en un periodo determinado. El enfoque de políticas no implica dispersar la energía entre un conjunto de temas variados, sino más bien tratar los problemas fundamentales que surgen de la adaptación del hombre a la sociedad. (Lasswell, 1992)

La discusión de dichas Ciencias de las Políticas, evolucionó hasta el grado de plantear, a partir de la comparación estructural entre Ciencias Duras y Ciencias Blandas, un grado de rigor metodológico sumamente alto, que permitió fortalecer los paradigmas de investigación social en acción de gobierno y toma de decisiones, particularmente en lo relativo a la seguridad, planteada por el mismo Laswell, con el propósito de lograr un óptimo decisional. Dicha fortalezca metodológica, fue sugerida también por Dror (1992), quien sostuvo que se deben generar diseños de investigación que incluyan la experimentación como un componente fundamental en la construcción de una decisión. No obstante, es importante resaltar que Dror y Laswell toman distancia en el sentido que las decisiones en seguridad implican un mínimo de experimentación, en comparación con otros espacios sectoriales.

En cuanto a definiciones puras de Política Pública, podemos encontrar las sugeridas por Heclo y Widavsky, quienes la caracterizan como "[...] una acción gubernamental dirigida hacia el logro de objetivos fuera de ella misma”. Por su parte, Mény y Thoenig (Roth, 2007), la definen como "la acción de las autoridades públicas en el seno de la sociedad", especialmente enfocadas a la seguridad, entendida esta como un bien público y como un servicio de policía.

Según Dubnick, por Política Pública ha de entenderse "las acciones gubernamentales, o sea, lo que los gobiernos dicen y hacen en relación con un problema o una controversia” (Dubnick y Bardes, 1983). Esta definición limita las discusiones sobre seguridad, pues parte del hecho de la existencia de una seguridad objetiva y una percepción de seguridad o seguridad subjetiva, la cual sería en última instancia, el motor de la decisión pública, abriendo así la puerta a decisiones erróneas. Para Hogwood, el término se refiere a un proceso que "[...] haya sido generado o por lo menos tratado al interior de un marco de procedimientos, de influencias y de organizaciones 
gubernamentales" (Roth, 2007), de manera que a juicio de este último autor, se puede inferir la necesidad de construir la política pública de seguridad y defensa de un país a partir de un diálogo multisectorial, diferenciando muy bien los sectores sociales que deben tomar parte en ella, así como las relaciones existentes entre dichos sectores, con el propósito de privilegiar el control civil sobre el militar.

Por su parte, Roth (2007, p. 27) propone que se pueden considerar al menos cuatro elementos centrales que permiten identificar la existencia de una Política Pública, incluyendo las políticas públicas de seguridad, a saber:

- Implicación del gobierno

- Percepción de problemas

- Definiciones de objetivos

- El proceso de adopción en sí mismo

En ese sentido, el mismo autor delimita la existencia de una Política Pública, siempre y cuando existan "instituciones estatales [que] asuman total o parcialmente la tarea de alcanzar objetivos estimados como deseables o necesarios, por medio de un proceso destinado a cambiar un estado de las cosas percibido como problemático" (Roth, 2007). De la definición propuesta por Roth se puede resaltar el hecho de que una política pública de seguridad debe contar con la condición sine qua non del monopolio de la fuerza y la violencia legítima por parte del Estado, de manera que se contaría permanentemente con un entramado institucional que asumiría la tarea de la supervivencia de los individuos y las demás instituciones.

Así, Roth (2007) enfatiza el sentido humano de una política pública cuando plantea que "la acción gubernamental se desarrolla para y a través de actores sociales, [pues] [s]on seres humanos quienes la conciben, la deciden y la implementan, e igualmente [son] los destinatarios de ella, directa o indirectamente".

Finalmente, Roth (s.f.) afirma que una Política Pública es aquel:

"[...] conjunto conformado por uno o varios objetivos colectivos considerados necesarios o deseables y por medios y acciones que son trazados, por lo menos parcialmente, por una institución u organización gubernamental con la finalidad de orientar el comportamiento de actores individuales o colectivos para modificar una situación percibida como insatisfactoria o problemática.

$\mathrm{Al}$ respecto resulta conveniente resaltar las reflexiones propuestas por Alejo Vargas, quien parte del hecho de considerar que una política pública no es una decisión asilada sino un conjunto de tomas de posición que involucran una o varias instituciones aisladas. Así, Vargas (1999, p. 58) plantea que las políticas públicas significan la materialización de las decisiones tomadas en términos de acciones que producen resultados sobre la situación problemática y los actores involucrados en la misma. 
Esto sugiere como una necesidad fundamental identificar el plano de problemas sociales y situaciones socialmente problemáticas como elemento central de la naturaleza de las Políticas Públicas. En ese sentido, una política pública se plantea como la continuación de un proceso que parte de la identificación de problemas sociales y continúa estableciendo los marcos de solución de manera definitiva, concertada o transitoria. De manera que resulta indispensable establecer un marco de partida, comprensivo de los problemas sociales, las necesidades, carencias y demandas de la sociedad en su conjunto o de sectores sociales particulares. Adicionalmente, esas situaciones socialmente problemáticas pueden ser definidas como aquellas en las cuales la sociedad de manera mayoritaria percibe un problema como relevante y considera que el régimen político debe entrar a enfrentarlo a través de políticas públicas (Ozslak y O’Donnell, 1981).

La definición que Alejo Vargas da al concepto de políticas públicas, lleva precisamente implícitas estas tensiones entre los problemas sociales y la necesidad de su trámite. Así, son definidas por este autor, como "el conjunto de sucesivas iniciativas, decisiones y acciones del régimen político frente a situaciones socialmente problemáticas y que buscan la resolución de las mismas o llevarlas a niveles manejables" (Vargas, 1999, p. 56). Vargas establece claramente que las Políticas Públicas son pues, una herramienta de gestión a problemas sociales sin que sean un paradigma definitorio de acción política, ya que acepta que no necesariamente, deben solucionar completamente un problema social y que además se fundamenta en incertidumbres, a pesar de considerar que "el objeto de cualquier propuesta de política es controlar y dirigir cursos futuros de acción, que es la única acción sujeta a control”.

Cabe resaltar que un elemento característico en los trabajos de Vargas es la tipología que hace sobre las políticas públicas, desde diferentes paradigmas teóricos. Esta herramienta facilita la adopción de diversos modelos, dependiendo de la situación que se debe analizar, o de la problemática social evidente (Ozslak y O’Donnell, 1981, pp. 64-69). Así, los paradigmas teóricos planteados son fundamentalmente seis:

1. Enfoque Neo Marxista: Donde se delimitan las políticas públicas a través de funciones asociadas a los Estados capitalistas. En ese sentido, se proponen dos tipos:
a. Políticas Públicas de Acumulación
b. Políticas Públicas de legitimación

2. Enfoque Estructuralista: Donde el marco de las políticas públicas es definido por los componentes e intereses de una estructura. Este enfoque distingue tres categorías:
a. Políticas Públicas Hegemónicas
b. Políticas Públicas Transaccionales
c. Políticas Públicas de Dominación 
3. Enfoque Socio Económico: Propuesta desarrollada por Fernando Rezende, donde se analiza la Política Pública en función del gasto estatal. Genera dos grandes tipos:
a. Políticas Públicas Promocionales
b. Políticas Públicas Correctivas

4. Enfoque sectorial: Propuesta por Donald Watson, donde los sectores se condicionan mutuamente y se encuentran en una situación de interrelación constante:
a. Política de Defensa Nacional
b. Política Exterior
c. Política Económica
d. Política social

5. La propuesta de Jorge Vianna Monteiro: la cual clasifica las políticas públicas según la perspectiva que más las afecte. Las perspectivas identificadas son: Jurisdicción político administrativa
a. Clientelas
b. Según el área administrativa

6. La tipología de Lowi: Donde se afirma que toda política pública traduce una voluntad manifiesta o implícita del gobernante, de influenciar la conducta o la acción de los individuos, ya sea directamente o indirectamente. A partir de estas posibles formas de interacción entre el gobernante y los ciudadanos, se establecen las siguientes categorías:
a. Políticas Públicas distributivas
b. Políticas Públicas de gasto público o de infraestructura
c. Políticas públicas redistributivas
d. Políticas públicas reguladoras o reglamentarias

Finalmente, tanto para Vargas, Roth, Jones o Meny la política pública debe ser un proceso en sí mismo. Además, todos coinciden en la necesidad de identificar un ciclo de acción de los mecanismos que enmarcan las políticas públicas. Así, básicamente, los autores identifican fases de inicio o de identificación del problema, de formulación de soluciones o de toma de decisiones, de implementación y de evaluación.

A partir de este marco de análisis, se entiende que las políticas públicas, incluidas las de seguridad y defensa, son un proceso de toma de decisiones y acciones desde lo público, que entienden la seguridad como la necesidad pública más apremiante y que debe ser contenida en una estructura institucional, con clara diferencia entre sus componentes, de manera que se garantice el control civil. Partiendo de esta definición, se procederá a analizar los estudios de caso de México y Brasil. 


\section{Política pública de seguridad y defensa mexicana: la inclusión de lo militar en la seguridad federal y la contrainsurgencia}

\section{De los documentos rectores}

Los documentos rectores, donde se definen los lineamientos de seguridad y defensa en México son, por un lado, Constitución Política de los Estados Unidos Mexicanos en sus artículos 10, 16 y particularmente el artículo 21, donde se especifica que:

La seguridad pública es una función a cargo de la Federación, el Distrito Federal, los Estados y los Municipios, que comprende la prevención de los delitos; la investigación y persecución para hacerla efectiva, así como la sanción de las infracciones administrativas, en los términos de la ley, en las respectivas competencias que esta Constitución señala.

La actuación de las instituciones de seguridad pública se regirá por los principios de legalidad, objetividad, eficiencia, profesionalismo, honradez y respeto a los derechos humanos reconocidos en esta Constitución. Las instituciones de seguridad pública serán de carácter civil, disciplinado y profesional. El Ministerio Público y las instituciones policiales de los tres órdenes de gobierno deberán coordinarse entre sí para cumplir los objetivos de la seguridad pública y conformarán el Sistema Nacional de Seguridad Pública, que estará sujeto a las siguientes bases mínimas:

a) La regulación de la selección, ingreso, formación, permanencia, evaluación, reconocimiento y certificación de los integrantes de las instituciones de seguridad pública. La operación y desarrollo de estas acciones será competencia de la Federación, el Distrito Federal, los Estados y los municipios en el ámbito de sus respectivas atribuciones.

b) El establecimiento de las bases de datos criminalísticos y de personal para las instituciones de seguridad pública. Ninguna persona podrá ingresar a las instituciones de seguridad pública si no ha sido debidamente certificado y registrado en el sistema.

c) La formulación de políticas públicas tendientes a prevenir la comisión de delitos.

d) Se determinará la participación de la comunidad que coadyuvará, entre otros, en los procesos de evaluación de las políticas de prevención del delito así como de las instituciones de seguridad pública.

e) Los fondos de ayuda federal para la seguridad pública, a nivel nacional serán aportados a las entidades federativas y municipios para ser destinados exclusivamente a estos fines. (UNIÓN, 2009)

También el artículo 73, sobre las facultades del Congreso, se estipula que son funciones de ese órgano colegiado:

[...] numeral XXIII: [Para] expedir leyes que establezcan las bases de coordinación entre la Federación, el Distrito Federal, los Estados y los Municipios, así como para establecer y 
organizar a las instituciones de seguridad pública en materia federal, de conformidad con lo establecido en el artículo 21 de esta Constitución"

y el numeral "XXIX-M [Para] expedir leyes en materia de seguridad nacional, estableciendo los requisitos y límites a las investigaciones correspondientes”. (UNIÓN, 2009)

El artículo 89 sobre las facultades del presidente, establece también directrices claras en ese sentido, pues se encuentran entre sus funciones:

IV. Nombrar, con aprobación del Senado, los Coroneles y demás oficiales superiores del Ejército, Armada y Fuerza Aérea Nacionales, y los empleados superiores de Hacienda.

V. Nombrar a los demás oficiales del Ejército, Armada y Fuerza Aérea Nacionales, con arreglo a las leyes.

VI. Preservar la seguridad nacional, en los términos de la ley respectiva, y disponer de la totalidad de la Fuerza Armada permanente o sea del Ejército, de la Armada y de la Fuerza Aérea para la seguridad interior y defensa exterior de la Federación.

VII. Disponer de la Guardia Nacional para los mismos objetos, en los términos que previene la fracción IV del artículo 76.

VIII. Declarar la guerra en nombre de los Estados Unidos Mexicanos, previa ley del Congreso de la Unión. (UNIÓN, 2009)

Por otro lado, se tiene como documento rector adicional a la Constitución Política arriba referenciada, la Ley de Seguridad Nacional del 31 de enero de 2005, reglamentada para el periodo 2009-2012 por el Programa para la Seguridad Nacional. Esta ley establece las directrices plenas de la política pública de seguridad y defensa, establece alcances e instituciones comprometidas. La Ley de Seguridad Nacional también define a la seguridad nacional -e incluso al Sistema de Seguridad Nacional- como

[...] las acciones destinadas de manera inmediata y directa a mantener la integridad, estabilidad y permanencia del Estado Mexicano que conlleven a:

- Proteger al país frente a riesgos y amenazas.

- Preservar la soberanía, independencia, territorio y la unidad de la federación.

- Mantener el orden constitucional y fortalecer las instituciones democráticas de gobierno.

- Defender al país frente a otros Estados o sujetos de derecho internacional.

- Preservar el régimen democrático fundado en el desarrollo social, económico y político. (Ley de Seguridad Nacional, 2005) 
De la estructura de seguridad y defensa mexicana

\section{a. Del sistema SEDENA - SEMAR}

El sistema de defensa mexicano se basa en dos Secretarías de Estado. Por un lado, la Secretaría de la Defensa Nacional SEDENA y por otro la Secretaría de Marina SEMAR. Adicionalmente existe un Estado Mayor presidencial que está adscrito a la presidencia de la república pero que depende en lo funcional de la SEDENA. La SEDENA, al excluir a la Armada, está compuesta solo por la estructura del Ejército y de la Fuerza Aérea Mexicana. Como puntos relevantes, tanto la SEDENA como la SEMAR son relativamente autónomas para diseñar y ejecutar su propia política de defensa (FESCOL, 2009 noviembre). Por otro lado, en la estructura de seguridad y defensa mexicana no existe el concepto de armas combinadas ni ejercicios conjuntos, como tampoco existe un Estado Mayor conjunto (Figura 1) (FESCOL, 2009 noviembre).

La SEDENA, en función de la seguridad interior, tiene un despliegue en todo el territorio mexicano compuesto por (FESCOL, 2009 noviembre):

- 12 regiones y 46 zonas militares (Ejército) y 3 regiones aéreas

- 18 bases aéreas y 27 escuadrones de combate

- 6 Unidades básicas tipo división

- 123 cuerpos de infantería

- 41 cuerpos de caballería

- 16 cuerpos de artillería

- 14 batallones de fuerzas especiales

- 3 batallones de ingenieros

- 3 batallones de policía militar

\section{Figura No 1: Sistema de Defensa en México: relación de mando}

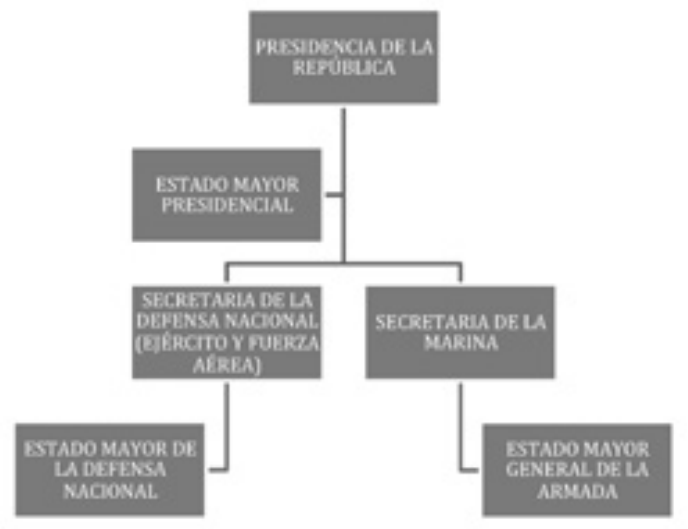

Fuente: Programa de Cooperación en Seguridad Regional FESCOL. Policy Paper No 27. Nov. 2009. 
La SEMAR, por su parte, está compuesta por (FESCOL, 2009 noviembre):

- 8 regiones navales

- 14 zonas navales

- 11 sectores

- 7 subsectores

- 27 apostaderos navales

- 30 batallones de infantería de marina

- 1 batallón de fusileros paracaidistas

- 1 batallón de guardia presidencial

Esta estructura, no obstante su tamaño (Tabla 2) tiene una composición ineficiente del gasto nacional en defensa. En primer lugar, el gasto solo corresponde a $0.4 \%$ del PIB en promedio durante la década actual de 2001 a 2009 (Figura 3), siendo este destinado en su mayoría al pago de oficiales, donde casi 90\% del presupuesto asignado se dirige a pago de salarios y prestaciones sociales (Secretaria de Hacienda y Crédito Público, 2010). Si se compara con el resto de la región latinoamericana, los resultados que se encuentran nos indican que el gasto en defensa y seguridad mexicano, sin embargo, es uno de los más bajos, como porcentaje del PIB dentro de la región (Ver cuadro 4).

Tabla No. 1 Personal Fuerzas Armadas Mexicanas 2008

\begin{tabular}{|l|l|l|l|l|l|}
\hline \multicolumn{1}{|c|}{ SEDENA } & Personal & \% del total & \multicolumn{1}{c|}{ SEMAR } & Personal & \% del total \\
\hline Generales & 537 & 0.3 & Almirantes & 221 & 0.4 \\
\hline Jefes & 5364 & 2.7 & Capitanes & 1713 & 3.1 \\
\hline Oficiales & 30110 & 15.1 & Oficiales & 12586 & 22.5 \\
\hline Tropa & 162686 & 81.5 & Clases & 40378 & 72.2 \\
\hline Otros & 848 & 0.4 & I M & 1063 & 1.9 \\
\hline Total & 199545 & 100 & Total & 55961 & 100 \\
\hline
\end{tabular}

Fuente: Sergio Aguayo. México todo en cifras 2008. Citado en: Programa de Cooperación en Seguridad Regional FESCOL. Policy Paper No 27. Nov. 2009

Esta distribución inadecuada ha hecho que no se invierta en renovación de equipo militar, aéreo o naval y que se facilite la deserción de cuadros de mando, en su mayoría sub oficiales, que pueden engrosar las filas de organizaciones criminales.

La problemática de la eficiencia del gasto militar mexicano se sostiene en la gran autonomía que gozan los militares en el manejo de recursos humanos y financieros. Tal y como lo expone FESCOL, esta situación se convierte en un rezago en materia de transparencia y es una tarea pendiente de la democracia mexicana (FESCOL, 2009 noviembre). 
Tabla No. 2 Porcentaje del Presupuesto de las Fuerzas Armadas Mexicanas con Relación al PIB 2001-2008

\begin{tabular}{|l|l|l|l|l|l|l|l|l|}
\hline AÑOS & $\mathbf{2 0 0 1}$ & $\mathbf{2 0 0 2}$ & $\mathbf{2 0 0 3}$ & $\mathbf{2 0 0 4}$ & $\mathbf{2 0 0 5}$ & $\mathbf{2 0 0 6}$ & $\mathbf{2 0 0 7}$ & $\mathbf{2 0 0 8}$ \\
\hline SEDENA & 0.39 & 0.36 & 0.33 & 0.30 & 0.29 & 0.28 & 0.34 & 0.29 \\
\hline SEMAR & 0.15 & 0.14 & 0.13 & 0.11 & 0.10 & 0.10 & 0.12 & 0.11 \\
\hline TOTAL & 0.54 & 0.50 & 0.46 & 0.41 & 0.39 & 0.39 & 0.46 & 0.40 \\
\hline
\end{tabular}

Fuente: Programa de Cooperación en Seguridad Regional FESCOL. Policy Paper No 27. Nov. 2009

\section{Figura No 2 Comparación del Gasto en Defensa de la Región y Gasto como Porcentaje del PIB.}

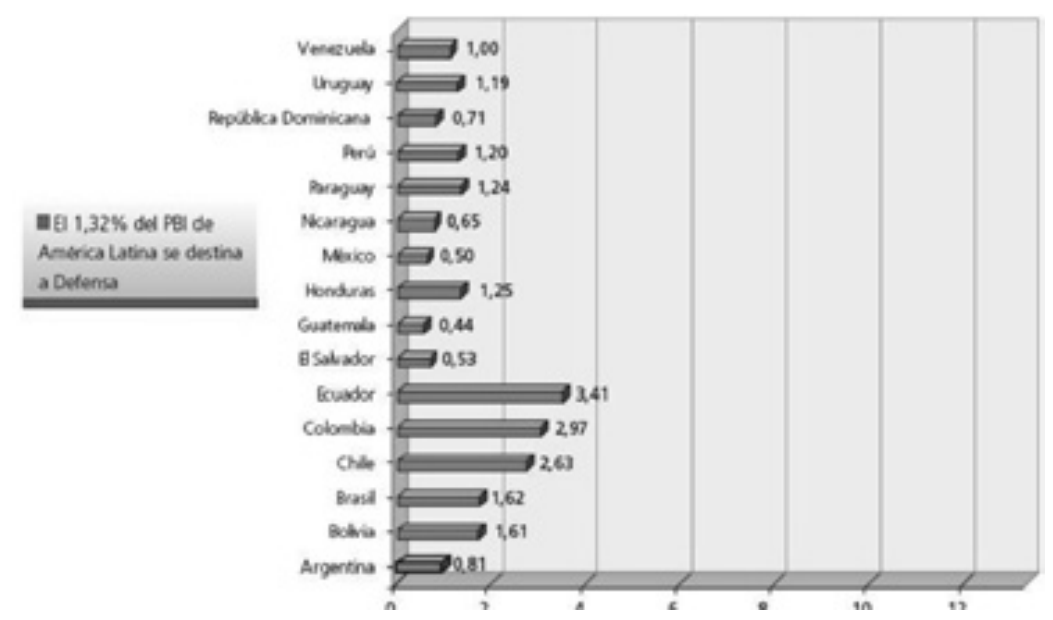

Fuente: Estadísticas de presupuesto en seguridad y defensa. RESDAL. www.resdal.org.

\section{b. Del Sistema de Seguridad Nacional}

El Sistema de Seguridad Nacional es encabezado por el Presidente de la República y se integra por las dependencias que forman parte del Consejo de Seguridad Nacional (CSN), las unidades de la Administración Pública Federal declaradas como instancias de Seguridad Nacional y por las autoridades estatales y municipales que colaboran con el Gobierno Federal a preservar la Seguridad de la Nación (Portillo, 2002).

Por el lado del Consejo de Seguridad Nacional, el CSN es una instancia deliberativa encabezada por el Presidente de la República, cuya finalidad es la de establecer y articular las acciones y políticas en materia de Seguridad Nacional. Por el lado de las instancias de Seguridad Nacional, estas son unidades administrativas de la Administración Pública Federal reconocidas por el Consejo de Seguridad Nacional CSN como parte del Sistema de Seguridad Nacional con el objeto de incorporarlas a las estrategias, acciones y políticas encaminadas a preservar la seguridad del país (Centro de Investigación en Seguridad Nacional , 2010) 
Tales instancias pueden ser agrupadas en las siguientes Secretarías (Centro de Investigación en Seguridad Nacional , 2010):

- Secretaría de Gobernación Instituto Nacional de Migración (INAMI); Coordinación General de Protección Civil y Secretaría Técnica de Coordinación para la Implementación del Sistema de Justicia Penal.

- Secretaría de Comunicaciones y Transportes: Oficina del Secretario; Subsecretaría de Transporte; Subsecretaría de Comunicaciones; Unidad de la Red Privada del Gobierno Federal; Unidad de Asuntos Jurídicos; Dirección General de Aeronáutica Civil; Dirección General de Autotransporte Federal; Dirección General de Transporte Ferroviario y Multimodal; Dirección General de Telecomunicaciones y Radiodifusión; Dirección General de Marina Mercante; Coordinación General de Puertos y Marina Mercante, y Servicios de Navegación en el Espacio Aéreo Mexicano.

- Secretaría de Hacienda y Crédito Público: Oficina del Secretario; Subsecretaría de Hacienda y Crédito Público (Unidad de Planeación Económica de la Hacienda Pública y Unidad de Crédito Público); Unidad de Inteligencia Financiera; así como la Procuraduría Fiscal de la Federación (Subprocuraduría Fiscal Federal de Legislación y Consulta, Subprocuraduría Fiscal Federal de Asuntos Financieros, Subprocuraduría Fiscal Federal de Amparos y Subprocuraduría Fiscal Federal de Investigaciones).

- Secretaría de la Función Pública: Dirección General de Información e Integración.

- Secretaría de Relaciones Exteriores: Dirección General de Protección a Mexicanos en el Exterior, Dirección General de Servicios Consulares, Dirección General de Delegaciones y Dirección General de Asuntos Jurídicos.

\section{De las características de la política pública de seguridad y defensa mexicana}

\section{a. Definiciones de Seguridad Nacional y Seguridad Pública}

La política pública de seguridad y defensa mexicana enfrenta inicialmente un problema de definición y de delimitación apremiante. Por un lado, la Constitución Política habla de Seguridad Pública, mientras que el Programa para la Seguridad Nacional 2009-2012, al igual que la Ley de Seguridad Nacional habla de Seguridad Nacional. Estos dos conceptos no pueden interpretarse como sinónimos, pues cada uno enmarca de una manera diferente las responsabilidades públicas a la hora de enfrentar amenazas a la seguridad. De acuerdo con la Constitución, la Seguridad Pública es una función concurrente a cargo de los tres niveles de gobierno. Comprende la prevención, investigación y persecución para hacerla efectiva, así como la sanción de las infracciones administrativas (UNIÓN, 2009).

La Seguridad Pública puede convertirse en asunto de Seguridad Nacional en el grado en que la manifestación del fenómeno criminal represente un riesgo para la integridad, estabilidad y permanencia del Estado, para el orden constitucional, las instituciones 
democráticas fundadas en el desarrollo social, económico y político, y en el grado en que constituyan un obstáculo para que las autoridades actúen contra la delincuencia organizada. De hecho, el Programa para la Seguridad Nacional (Centro de Investigación en Seguridad Nacional , 2010), la define, en concordancia con el artículo 3 de la Ley de Seguridad Nacional (Portillo, 2002), específicamente como:

Las acciones destinadas de manera inmediata y directa a mantener la integridad, estabilidad y permanencia del Estado Mexicano, que conlleven a:

I. La protección de la nación mexicana frente a las amenazas y riesgos que enfrente nuestro país;

II. La preservación de la soberanía e independencia nacionales y la defensa del territorio;

III. El mantenimiento del orden constitucional y el fortalecimiento de las instituciones democráticas de gobierno;

IV. El mantenimiento de la unidad de las partes integrantes de la Federación señaladas en el artículo 43 de la Constitución Política de los Estados Unidos Mexicanos;

V. La defensa legítima del Estado Mexicano respecto de otros Estados o sujetos de derecho internacional, $y$

VI. La preservación de la democracia, fundada en el desarrollo económico, social y político del país y sus habitantes.”

Para el Centro de Investigación y Seguridad Nacional mexicano, las diferencias entre los dos conceptos se pueden sistematizar en función de sus objetivos, instrumentos y autoridades responsables. Así, lo que se percibe de esta diferenciación es que el concepto de Seguridad Nacional se enmarca en un sentido estratégico, de responsabilidad inter agencial son un gran esfuerzo militar, mientras que el concepto de Seguridad Pública es un tanto más limitado, que toca instancias locales y que llega a tener visos o matices policiales (Tabla 5)

Tabla No. 3 Distinción entre Seguridad Nacional y Seguridad Pública

\begin{tabular}{|l|l|l|}
\hline & \multicolumn{1}{|c|}{ Seguridad Nacional } & \multicolumn{1}{c|}{ Seguridad Pública } \\
\hline Objetivos & $\begin{array}{l}\text { Tiene como propósito mantener la } \\
\text { integridad, estabilidad y permanencia } \\
\text { del Estado Mexicano. }\end{array}$ & $\begin{array}{l}\text { Su objetivo es salvaguardar la } \\
\text { integridad y derechos de las personas, } \\
\text { así como preservar las libertades, el } \\
\text { orden y la paz públicos. }\end{array}$ \\
\hline Instrumentos & $\begin{array}{l}\text { Sus instrumentos son la operación } \\
\text { de tareas de inteligencia y } \\
\text { contrainteligencia para proponer } \\
\text { medidas de prevención, disuasión, } \\
\text { contención o neutralización de } \\
\text { riesgos o amenazas. }\end{array}$ & $\begin{array}{l}\text { Sus instrumentos son la prevención, } \\
\text { persecución, sanción de las } \\
\text { infracciones, así como la reinserción } \\
\text { social de los delincuentes e } \\
\text { infractores. }\end{array}$ \\
\hline
\end{tabular}




\begin{tabular}{|l|l|l|}
\hline & \multicolumn{1}{|c|}{ Seguridad Nacional } & \multicolumn{1}{c|}{ Seguridad Pública } \\
\hline \multirow{4}{*}{ Autoridades } & La Seguridad Nacional es materia & La Seguridad Pública es una materia \\
responsables & federal. El Gobierno de la República & concurrente en la que los tres \\
& establece mecanismos de cooperación & niveles de gobierno comparten la \\
& con las autoridades estatales y & responsabilidad de la misma en los \\
municipales. & ámbitos de su competencia. \\
\hline
\end{tabular}

Fuente: CISEN. http://www.cisen.gob.mx/.

\section{b. Identificación de riesgos y amenazas en la política pública de Seguridad y Defensa Mexicana}

La diferenciación de estos dos conceptos aparece delimitada en la Ley de Seguridad Nacional y en el Programa de Seguridad Nacional 2009-2012. Esta diferencia puede pensarse en tres dimensiones, en primer lugar en cuanto la definición, en segundo lugar en cuanto efectos y en tercer lugar, en cuanto a orientación.

De esta manera, la definición de amenaza se encuentra en la Ley de Seguridad Nacional en el capítulo V, determinando permanentemente amenazas como (Portillo, 2002):

I. Actos tendentes a consumar espionaje, sabotaje, terrorismo, rebelión, traición a la patria, genocidio, en contra de los Estados Unidos Mexicanos dentro del territorio nacional;

II. Actos de interferencia extranjera en los asuntos nacionales que puedan implicar una afectación al Estado Mexicano;

III. Actos que impidan a las autoridades actuar contra la delincuencia organizada;

IV. Actos tendentes a quebrantar la unidad de las partes integrantes de la Federación, señaladas en el artículo 43 de la Constitución Política de los Estados Unidos Mexicanos;

V. Actos tendentes a obstaculizar o bloquear operaciones militares o navales contra la delincuencia organizada;

VI. Actos en contra de la seguridad de la aviación;

VII. Actos que atenten en contra del personal diplomático;

VIII. Todo acto tendente a consumar el tráfico ilegal de materiales nucleares, de armas químicas, biológicas y convencionales de destrucción masiva;

IX. Actos ilícitos en contra de la navegación marítima;

$\mathrm{X}$. Todo acto de financiamiento de acciones y organizaciones terroristas;

XI. Actos tendentes a obstaculizar o bloquear actividades de inteligencia o contrainteligencia, y

XII. Actos tendentes a destruir o inhabilitar la infraestructura de carácter estratégico o indispensable para la provisión de bienes o servicios públicos.

En síntesis, un análisis de las amenazas definidas sugieren que la variable que permite reunir a las amenazas es precisamente la existencia de amenazas asimétricas, donde 
el narcotráfico, el terrorismo y la guerra red aparecen como unas de las principales preocupaciones (El Universal, 2009).

A diferencia de la definición de amenaza, la definición de riesgo es dada por la Agencia Nacional de Riesgos tomando en cuenta el Plan Nacional de Desarrollo y el Programa para la Seguridad Nacional. Dichos riesgos, en comparación con la tipificación de las amenazas, no son permanentes, sino que se actualizan en función de la coyuntura.

En segundo lugar, en cuanto a los efectos de cada concepto, la amenaza determina todas las acciones legales y políticas en materia de seguridad nacional e incluso prevé un recorte temporal en derechos civiles permitiendo incluso interceptación en las comunicaciones de los ciudadanos. Los riesgos por su lado, orientan la ejecución de la política de seguridad nacional desde un punto de vista estratégico sobre la probable ocurrencia de hechos que tengan la capacidad de vulnerar la seguridad nacional

En tercer lugar, en relación con la orientación que la política de seguridad y defensa mexicana debe tomar en función de las amenazas, esta se constituye en un mecanismo de confrontación de tal amenaza con el fin de neutralizarla o desactivarla. El riesgo a diferencia, busca generar estrategias de prevención y disuasión fundamentalmente. Así, los riesgos a la seguridad nacional, dentro de la política pública de seguridad y defensa en México, puede definirse como "una condición interna o externa generada por situaciones políticas, económicas, sociales o agentes no estatales, así como por desastres naturales, de origen humano o epidemias, que sin tener carácter de amenazas pudieran poner en entredicho el desarrollo nacional" (Centro de Investigación en Seguridad Nacional , 2010).

\section{De la participación de las Fuerzas Armadas mexicanas en la respuesta para enfrentar las amenazas}

A partir del gobierno del presidente Felipe Calderón, las fuerzas armadas mexicanas entraron de lleno en el combate a las amenazas internas, fundamentalmente el narcotráfico. Este giro, de tener unas fuerzas armadas enfocadas al cuidado fronterizo y re direccionarlas hacia el combate interno asimétrico e irregular, se debió a varios factores. En primer lugar, el poder de fuego cada vez mayor de las agrupaciones armadas asociadas al narcotráfico que sobrepasaban la capacidad de las policías locales de manera que solo en 2008 se produjeron 6.289 muertes violentas asociadas al narcotráfico ${ }^{1}$.

En segundo lugar, al grado de corrupción de estas policías locales y de las demás instituciones de seguridad pública, las cuales fueron permeadas por sectores criminales generando cooperación de estas estructuras con la ilegalidad o simplemente la

\footnotetext{
${ }^{1}$ Decreto por el que se reforma el acuerdo que crea el Cuerpo Especial del Ejército y fuerza aérea denominado Cuerpo de Fuerzas de Apoyo federal. Diario Oficial de la Federación. 17 de Septiembre de 2009.
} 
sustracción de sus miembros hacia organizaciones criminales, siendo el caso de Los Zetas el más conocido. Sobre este punto, resulta interesante el hecho que luego de la inclusión de militares en la guerra interna, alguna porción significativa de los generales y demás comandantes militares fueron delegados para dirigir otras instituciones de control de seguridad, fundamentalmente cuerpos locales de policía y otras agencias locales de seguridad (Zermeño, 2009 febrero 15).

En tercer lugar, al prestigio que gozan las fuerzas militares mexicanas, las cuales se pueden ubicar dentro de las tres entidades gubernamentales que más confianza tienen dentro de la sociedad. De hecho, en su momento, ocho de cada diez mexicanos estuvieron de acuerdo con la decisión presidencial para utilizarlas en el combate al narcotráfico. Así, se incrementó notablemente el número de efectivos de las fuerzas armadas dedicadas al combate interno, pasando de 9.970 a más de 45.00o efectivos (Aguayo, 2008)

Esta inclusión, debido al apoyo popular que tienen las fuerzas militares mexicanas, no generó mayores suspicacias ni reclamos por parte de la población civil, ni se abrió la discusión sobre la necesidad del control de lo civil sobre lo militar. En sí, la sociedad mexicana ve con buenos ojos la institución militar y no la entiende como un riesgo a al estabilidad democrática sino al contrario, como un garante. Por otro lado, la construcción de amenazas y retos dentro de los documentos rectores de seguridad y defensa mexicanos, fueron construidos teniendo en cuenta amplios sectores sociales académicos y económicos, de manera que se da por hecho el control civil en la identificación de tareas y asignación de roles para las fuerzas armadas mexicanas (Benítez, 2008).

\section{Política pública de seguridad y defensa de Brasil: norte regional de una nueva concepción de contrainsurgencia}

\section{De las generalidades de la política pública de seguridad y defensa del Brasil}

La reorientación de la política pública de seguridad y defensa de Brasil hizo trámite desde el cambio mismo de régimen y la instauración del gobierno civil de Fernando Collor de Mello. Dicha estructura estuvo caracterizada durante los años de gobierno militar por seis componentes, los ministerios del Ejército, la Marina y la Aeronáutica, el Estado Mayor de las Fuerzas Armadas, la Casa Militar de la Presidencia de la República y el Servicio Nacional de Información. Con el cambio de régimen y la administración Collor de Mello, se suprimió el Servicio Nacional de Información y quedó la estructura reducida solo a cinco instancias. Como plantea Alcídes Acosta e Igor Calvet, esta estructura era el reflejo del dominio de las Fuerzas Armadas sobre el poder ejecutivo durante el régimen militar de manera que cada una de las fuerzas, Ejército, Armada y Fuerza Aérea, tuviera un peso específico propio y gozara adicionalmente de una amplia autonomía frente a la otra (Acosta, s.f.).

No obstante, este sistema, hijo del régimen militar, no respondía aún a las necesidades de transición, pues seguía siendo anacrónico y fue necesario realizar su ajuste. Dicho 
cambio se dio en 1996, cuando se adoptó la Política de Defensa Nacional PDN, bajo iniciativa del presidente Fernando Enrique Cardoso. Dicho documento se constituye en el documento rector de la política pública de seguridad y defensa brasileña desde entonces.

La política de Defensa Nacional fue el primer documento sectorial construido para servir de guía en materia de seguridad y defensa. Sin embargo, debido entre otras cosas a su mismo carácter inédito, la PDN se asoció, según Acosta y Calvet, menos a su contenido que a los objetivos necesarios o intereses fundamentales en materia de seguridad brasileña de inicios de la década de 1990 (Acosta, s.f.), tales como la "apertura de un camino hacia el ordenamiento institucional del área de seguridad”.

El diseño de la Política de Defensa Nacional fue sin embargo, no tan abierto y democrático como se hubiera podido esperar por tratarse de un documento base para la transición democrática de un país latinoamericano. Su elaboración fue cerrada y solo fue competencia del Departamento de Políticas y Articulación Institucional y un círculo selecto de académicos expertos en seguridad y defensa. Solo se realizaron algunos seminarios esporádicos que incluyeron a algunos de los mandos de las fuerzas armadas brasileñas.

En este ámbito restringido, el proceso de elaboración de la Política de Defensa Nacional encontró, según Costa y Calvet, una serie de obstáculos y dificultades entre los que se pueden enumerar diferencias a la hora de establecer acuerdos entre los intereses divergentes de las fuerzas armadas en su interior y la cultura política del sistema político del Brasil - la cual daba preferencia a la autonomía de las fuerzas armadas y a la autonomía que existía, desde el periodo militar, para que cada fuerza diseñara su propia política y directrices.

Pero tal vez, el principal problema de este primer documento de Política de Defensa Nacional fue el hecho de ser demasiado vago y genérico. De esta manera, no se delinearon intereses estratégicos creíbles y alcanzables ni se lograron establecer mecanismos iniciales de control civil sobre lo militar en ninguna de las tres fuerzas armadas brasileñas.

\section{La nueva Política de Defensa Nacional. El camino hacia el cambio}

En junio de 2005 se aprobó la nueva Política de Defensa Nacional intentando ser una solución a los vacíos y zonas grises de la Política anterior. Sus objetivos generales eran:

a. Definir el empleo y las funciones de las Fuerzas Armadas del Brasil

b. Definir la importancia de la seguridad en las relaciones exteriores de Brasil

c. Propender por el perfeccionamiento de la institucionalidad democrática y de transición

d. Instaurar una cultura de defensa en la sociedad brasileña

Sin embargo, esta política al parecer tampoco fue efectiva en un comienzo ni al interior ni en el ámbito internacional de Brasil, ámbito en el cual se quiere proyectar como potencia regional o potencia emergente. 


\section{a. Caracterización del ambiente internacional}

La PDN establece cuatro niveles de análisis del entorno internacional de Brasil, en primer lugar la estructura de poder vigente, en segundo lugar las relaciones inter estatales y el marco de la política exterior de Brasil, en tercer lugar, las formas de amenaza tradicional y en cuarto lugar, las nuevas formas de amenaza.

El primero de los aspectos es evidente en la PDN, al determinarlo como un ambiente de relativa estabilidad donde conflictos interestatales son cada vez más escasos e improbables. No obstante, la PDN aclara que frente a la ausencia de conflictos tradicionales, existen catalizadores de conflictos y nuevas guerras pueden aparecer en el panorama internacional. De esta manera, la PDN inicia su marco con la discusión fundamental de la importancia de preparar sus fuerzas en los conceptos de la guerra asimétrica, propia, según la PDN, de este sistema internacional.

Para el segundo de los aspectos centrado en las relaciones interestatales y la política exterior de Brasil, la preocupación que la PND evidencia en primer lugar y de la mano con el planteamiento anterior, es el de conflictos generados por recursos energéticos estratégicos y el control de dichas áreas. El dilema generado es que se recurra a una variable obsoleta como posibles diferendos limítrofes que dificulten el liderazgo de Brasil y generen inestabilidad regional.

El tercer aspecto se deriva precisamente del análisis anterior. Las amenazas tradicionales que se conciben en la PDN se encierran en mayor medida en el tema de diferendos limítrofes motivada por la escaza presencia y control de fronteras en algunas de las regiones del país. Este vacío de poder estatal sumado a las actividades ilegales que prosperan en zonas de frontera, vuelven un reto importante el cuidado fronterizo dentro de la lógica de la defensa nacional.

Finalmente, el último aspecto se relaciona con las nuevas amenazas. Es tal vez dentro de este aspecto, donde la PDN profundiza más, identificando elementos como los conflictos étnico religiosos, la exacerbación de nacionalismos, la fragmentación de los Estados, el terrorismo y la biopiratería como unas de las principales amenazas a la seguridad nacional de Brasil (Acosta, s.f.). Precisamente sobre este punto es que existe la principal novedad o aporte de la PDN en comparación con los intentos anteriores, consistente en la delimitación de dos áreas estratégicas como lo es el Atlántico Sur y la Amazonía Brasileña.

Tradicionalmente las preocupaciones militares de Brasil se concentraban en el espacio geoestratégico de la Amazonía y dejaba de lado otros espacios. La nueva PDN revitaliza la importancia en recursos que ofrece el Atlántico sur, así como la posibilidad de interconectar a Brasil con el África y Asia, además de asegurar una zona naval que estando vacía representa mayores problemas de vulnerabilidad que una zona fronteriza terrestre común. Tal y como lo afirman Costa y Calvet, el 75\% de la producción de gas 
y petróleo del Brasil, así como una gran biodiversidad marina y las principales rutas de navegación global hacia el sur, pasan por esta franja marítima.

La PDN además, complementa la acción de estos dos ejes plenamente delimitados con dos temas de orden interno, como lo son por un lado la defensa del espacio aéreo y el desarrollo de la industria de defensa. Aparte de estas dos preocupaciones, el interés de Brasil por acercarse a cuestiones globales lo proyectan a temas como el desarme multilateral, el terrorismo y la cooperación internacional.

A esta Política Nacional de Defensa de 2005, se le suma el proyecto de una nueva Estrategia de Defensa propuesta en 2008, como se indica en seguida, la cual representa la maximización del paradigma del desarrollo en las lógicas de la defensa.

\section{Criterios democráticos en la Política de Defensa Nacional de Brasil}

El avance entre los dos momentos de la Política de Defensa Nacional es notorio. La primera, apenas hija de la transición, era una construcción cerrada, limitada y poco ambiciosa, además de vaga y genérica, lo cual facilitaba la autonomía de las fuerzas en la formulación de las políticas de defensa.

La actual Política de Defensa Nacional es un poco más inclusiva y cuenta con espacios de debate por medio del control legislativo. En particular, los cambios más fundamentales giran además entorno a la identificación de los elementos del interés nacional sin entrar en la discusión específica de qué es riesgo y qué es amenaza. Este interés nacional delimitado permite definir con claridad las dos áreas estratégicas en la seguridad y defensa de Brasil, construyendo unos mecanismos de participación que incluye desde medioambientalistas hasta industriales.

Es posible afirmar, no obstante que la plena democracia aún está lejos dentro de la construcción de políticas públicas de seguridad en Brasil, que si bien la autonomía militar se ha recortado, el persistente celo relacionado con el conocimiento y la actividad militar se mantiene, convirtiéndose en un tema de cultura política a superar para poder completar un proceso real de transición democrática.

Adicionalmente, el impulso de las nuevas lógicas de la defensa en torno a las necesidades de consolidar el desarrollo del país en su nuevo objetivo de disputa del poder global, ha sido determinante en los nuevos objetivos de la política general brasileña, cuyas últimas administraciones han asumido un importante rol en la superación de la lógica unipolarizada derivada de la Guerra Fría y promueven una distribución del poder más difusa, en la que Brasil juega un papel preponderante a través del posicionamiento de algunos países como centros alternativos de poder, organizados en dos plataformas: (i) el IBSA: India, Brasil, y Sudáfrica; y (ii) el BRIC: Brasil, Rusia, India y China.

Adicionalmente, es importante tener presente en ese cambio de la política general y en consecuencia de la política de defensa, el rol de liderazgo de la República Brasileña 
en la promoción de la UNASUR y del Consejo Suramericano de Defensa. Todo ello con el objetivo de consolidar a Brasil como un gran poder en el Siglo XXI, lo cual deja como un gran saldo, la transformación del rol diplomático y militar de Estados Unidos en América Latina (Brands, 2010).

Los principales postulados de fortalecimiento brasileño son:

- Restablecer la estabilidad macroeconómica, a través de:

- La implementación de políticas económicas que remuevan los obstáculos a la inversión y así generar mayores márgenes de crecimiento.

- Proyectos de infraestructura que faciliten el comercio

- Reducir la pobreza

- Establecer una democracia activa

- Mediante, entre otros mecanismos, reformas políticas que mitiguen la corrupción $\mathrm{y}$ alienten el establecimiento y fortalecimiento de gobiernos y administraciones responsables.

- Lidiar de una manera efectiva con los insistentes problemas sociales

- A través de la implementación de políticas sociales que mejoren la calidad de la educación primaria y secundaria y que aseguren un mejor acceso a los servicios esenciales.

- A través de la adopción de medidas mixtas anti-crimen que reduzcan la inseguridad ciudadana.

\section{Conclusión}

El cuestionamiento sobre los diálogos de las políticas de seguridad y defensa de México y Brasil con los elementos propuestos desde la doctrina sobre la democratización de esta clase de políticas es una tarea permanente.

$\mathrm{Al}$ respecto Carlos Barrachina propone en Los libros blancos de la defensa como una reforma al sector, que los elementos característicos de políticas de defensa transparente e incluyente, deben ser por un lado "[un] documento de política en el que se expone el concepto de política del gobierno en el mediano plazo”, de acuerdo a los lineamientos del Consejo de Seguridad Hemisférica de la OEA. En segundo lugar, debe explicar la política y la doctrina de la defensa prevista (analizando la seguridad y el contexto interno), y mencionando la estrategia de reacción frente a los peligros contemplados.

En tercer lugar, debe agotar un amplio proceso de consultas dentro y fuera del gobierno y así lograr un consenso amplio. En cuarto lugar, debe explicar las funciones que cumplen las fuerzas armadas y la importancia de esas funciones en la sociedad. En quinto lugar debe detallar la capacidad de cada sistema de defensa, incluyendo una precisión en: (i) los medios con los que cuentan los ejércitos, (ii) las reglas básicas de operatividad y (iii) la estructura militar del sistema. Por último, debe expresar el presupuesto con el que se cuenta para la implantación de esa política. 
Del análisis de esos elementos en cada una de las políticas estudiadas, se concluye lo siguiente:

Tabla No. 4 Comparativo

\begin{tabular}{|c|c|c|}
\hline $\begin{array}{l}\text { CRITERIO DE } \\
\text { COMPARACIÓN }\end{array}$ & BRASIL & MÉXICO \\
\hline \multicolumn{3}{|c|}{ [Propuestos por Barrachina] } \\
\hline $\begin{array}{l}\text { Concepto de Política de } \\
\text { Gobierno }\end{array}$ & $\begin{array}{l}\text { Superación de la lógica } \\
\text { unipolarizada derivada de } \\
\text { la Guerra Fría hacia una } \\
\text { distribución del poder más } \\
\text { difusa } \\
\text { Y: Defensa para el } \\
\text { Desarrollo }\end{array}$ & $\begin{array}{l}\text { Conservación del Estado } \\
\text { Democrático y superación de } \\
\text { los focos del Conflicto }\end{array}$ \\
\hline Política y Doctrina de Defensa & $\begin{array}{l}\text { Defensa Nacional para el } \\
\text { Desarrollo Nacional }\end{array}$ & $\begin{array}{l}\text { Constitución: } \\
\text { Seguridad Pública y en } \\
\text { Ley y Programa: Seguridad } \\
\text { Nacional }\end{array}$ \\
\hline $\begin{array}{l}\text { Proceso de Consultas en su } \\
\text { construcción }\end{array}$ & $\begin{array}{l}\text { Comité Interministerial diseñó } \\
\text { proyecto, para que sea } \\
\text { resuelto por el Ejecutivo }\end{array}$ & $\begin{array}{l}\text { 'Ley de Seguridad Nacional', } \\
\text { discutida en el Congreso, y } \\
\text { reglamentada por el Ejecutivo } \\
\text { mediante el 'Programa para } \\
\text { la Seguridad Nacional 2009- } \\
2012^{\prime}\end{array}$ \\
\hline $\begin{array}{l}\text { Funciones de las Fuerzas } \\
\text { Armadas e importancia en la } \\
\text { sociedad }\end{array}$ & $\begin{array}{l}\text { Transformación de las } \\
\text { funciones tradicionales, bajo } \\
\text { lógicas más estratégicas, } \\
\text { de acuerdo a las nuevas } \\
\text { necesidades de la defensa. } \\
\text { [Son explicadas con algo de } \\
\text { detalle en la estrategia } \\
\text { de 2008] }\end{array}$ & $\begin{array}{l}\text { Enlista las tradicionales } \\
\text { asignadas por la Constitución } \\
\text { y la Ley }\end{array}$ \\
\hline $\begin{array}{l}\text { Capacidad de cada sistema de } \\
\text { defensa: } \\
\text { - Medios } \\
\text { - Reglas } \\
\text { - Capacidad de fuerza y } \\
\text { operativa } \\
\end{array}$ & No incluido & No incluido \\
\hline Presupuesto & No incluido & No incluido \\
\hline \multicolumn{3}{|c|}{ [Otros Criterios Identificados] } \\
\hline Mecanismos de definición & $\begin{array}{l}\text { - Política de Defensa Nacional } \\
\text { (PDN - 2005) } \\
\text { - Estrategia Nacional de } \\
\text { Defensa (Dic. 17, 2008) }\end{array}$ & $\begin{array}{l}\text { - Constitución Nacional } \\
\text { - 'Ley de Seguridad Nacional' } \\
\text { (2005) } \\
\text { - 'Programa para la Seguridad } \\
\text { Nacional 2009-2012' }\end{array}$ \\
\hline
\end{tabular}




\begin{tabular}{|c|c|c|}
\hline Objetivos & $\begin{array}{l}\text { - Reorganización de las } \\
\text { fuerzas armadas } \\
\text { - Reconstrucción de la } \\
\text { industria brasileña del } \\
\text { material de defensa } \\
\text { - Política de composición de } \\
\text { las FFAA (Unidad Nacional) } \\
\text { - Disuación, fortalecimiento } \\
\text { estatal e integración }\end{array}$ & $\begin{array}{l}\text { - Asegurar la defensa del } \\
\text { Estado, sus instituciones y la } \\
\text { soberanía nacional. } \\
\text { - Proteger al país frente a } \\
\text { riesgos y amenazas. } \\
\text { - Preservar la soberanía, } \\
\text { independencia, territorio y la } \\
\text { unidad de la federación. } \\
\text { - Mantener el orden } \\
\text { constitucional y fortalecer } \\
\text { las instituciones } \\
\text { democráticas de gobierno. } \\
\text { - Defender al país frente a } \\
\text { otros Estados o sujetos de } \\
\text { derecho internacional. } \\
\text { - Preservar el régimen } \\
\text { democrático fundado en el } \\
\text { desarrollo social, económico } \\
\text { y político". }\end{array}$ \\
\hline Estructura & $\begin{array}{l}\text { - Presidencia } \\
\text { - Ministerio de Defensa como } \\
\text { Estado Mayor Conjunto: } \\
\text { atado a un control político } \\
\text { constitucional } \\
\text { - Ejército - Armada - Fuerza } \\
\text { Aérea } \\
\text { - [Todas ellas con nuevos } \\
\text { rasgos] }\end{array}$ & $\begin{array}{l}\text { - Presidente } \\
\text { - Estado Mayor Presidencial } \\
\text { - Dos Secretarías: SEDENA y } \\
\text { SEMAR } \\
\text { - Ejército - Armada - Fuerza } \\
\text { Aérea }\end{array}$ \\
\hline Amenazas & $\begin{array}{l}\text { - Estructura del poder } \\
\text { vigente: Contexto } \\
\text { geopolítico } \\
\text { - Relaciones interestatales y } \\
\text { política exterior: Conflictos } \\
\text { generados por el control de } \\
\text { recursos estratégicos } \\
\text { - Amenazas tradicionales: } \\
\text { Diferendos limítrofes } \\
\text { - Nuevas amenazas: } \\
\text { Conflictos étnico } \\
\text { religiosos, la exacerbación } \\
\text { de nacionalismos, la } \\
\text { fragmentación de los } \\
\text { Estados, el terrorismo y la } \\
\text { biopiratería }\end{array}$ & $\begin{array}{l}\text { - Espionaje, sabotaje, } \\
\text { terrorismo, rebelión, traición } \\
\text { a la patria, genocidio } \\
\text { - Interferencia extranjera } \\
\text { - Delincuencia organizada } \\
\text { - Unidad } \\
\text { - Obstaculización de } \\
\text { operaciones militares } \\
\text { - Peligros nucleares } \\
\text { - Terrorismo } \\
\text { - Bloqueo de inteligencia y } \\
\text { contrainteligencia } \\
\text { - Deterioro de Infraestructura }\end{array}$ \\
\hline
\end{tabular}

Fuente: elaboración propia de los autores, sobre el modelo propuesto por Barrachina. 
En consecuencia podríamos afirmar que las políticas públicas de seguridad y defensa tanto de México como de Brasil, nos permiten encontrar dos escenarios complejos, interesantes y particularmente diferentes. Por un lado, la política de seguridad mexicana es un tanto más conservadora pues parte de la identificación de amenazas permanentes y la existencia de riesgos coyunturales. Por su parte la política de seguridad de Brasil, identifica en primer lugar nociones de interés nacional y sobre ella genera los lineamientos centrales de acción.

El sistema mexicano, en segundo lugar, ha superado en apariencia el tema democrático y de control civil de lo militar, de manera que el estamento militar es tremendamente reconocido y legitimado al interior de la sociedad y su uso para actividades internas no generan mayores preocupaciones. Brasil por su parte, no ha completado plenamente su transición hacia la democracia, y se constituye en un sistema de prevalencia de elementos de una sociedad militarizada. El control civil de lo militar en Brasil, es un escenario aún por lograr plenamente

Finalmente, las dos construcciones de seguridad y defensa identifican en sus amenazas, elementos propios de las nuevas amenazas del sistema internacional y prevén sus fuerzas para el combate de amenazas asimétricas. La recurrencia a esta proyección se sostiene en el hecho que tanto México como Brasil se ven como potencias al menos regionales, si no en ascenso, de manera que es necesario que intervengan en procesos de gran alcance del sistema internacional. El camino, sin embargo no se ha completado para ninguna de las dos políticas y sobretodo, en un sistema internacional guiado por la acción antiterrorista, la participación de dos sistemas, el mexicano y el brasileño, será de especial importancia.

\section{Referencias}

Acosta, C. (s.f.). La Política de defensa Brasileña: Diagnóstivos, propuestas y perspectivas para la cooperación regional. Programa de Cooperación en Seguridad Regional. Policy Paper no. 1o. Fundación Friedrich Ebert Stiftung FESCOL.

Aguayo, S. (2008). El almanaque Mexicano 2008. México: Ed. Aguilar.

Benítez, R. (2008). Las Fuerzas Armadas Mexicanas: desafíos y estructuras frente a la democracia. ATLAS Comparativo de la Defensa en América Latina (pp. 218231).Buenos Aires: RESDAL.

Brands, H. (2010, agosto). Dilemmas of Brazilian Grand Strategy. US: Strategic Studies Institute Monograph. Disponible en: http://www.strategicstudiesinstitute. army.mil/pdffiles/PUB1017.pdf

Centro de Investigación en Seguridad Nacional. (2010, octubre 20). Centro de Investigación en Seguridad Nacional. Disponible en: http://www.cisen.gob.mx. 
Dror, Y. (1992). Prolegómenos par las Ciencias Políticas. En L. Aguilar Estudio de Políticas Públicas (pp. 24-36). México: Grupo editorial Miguel Ángel Porrúa.

Dubnick, M. J., y Bardes, B. A. (1983). Thinking about public policy: A problem-solving approach. New York: John Wiley \& Sons Inc.

El Universal. (2009 febrero 27). 'El crimen es la mayor amenaza a los derechos humanos'. Entrevista a Felipe Calderón. El Universal.Disponible en: http://www. eluniversal.com.mx/nacion/166067.html

FESCOL (2009, noviembre). Programa de Cooperación en Seguridad Regional. Policy Paper No 27. Fundación Friedrich Ebert Stiftung FESCOL.

Laswell, H. (1992). La orientación hacia las políticas. En L. Aguilar, El estudio de las políticas públicas (pp. 39-74 ). México: Grupo Editorial Miguel Ángel Porrúa.

Ley de Seguridad Nacional. (2005). Diario Oficial de la Federación. México: Secretaría de Gobernación.

Ozslak, O., y O’Donnell, G. (1981). Estado y Políticas estatales: hacia una estrategia de investigación. Revista Administración y Desarrollo, (19).

Portillo, E. (2002). Hacia la Ley de Seguridad Nacional. Reflexiones para un ejercicio administrativo inédito. 15-16.

Roth, A. (2007). Políticas Públicas. Formulación, implementación y evaluación . Bogotá D.C.: Ediciones Aurora.

Roth, A. (s.f.). Etat et Politiques en Amérique Latina. Le cas des politiques environnementales en Colombie. Ginebra: Université de Genève.

Secretaria de Hacienda y Crédito Público. (2010, octubre 11). Orientación del gasto de la CEDENA y SEMAR, Presupuesto de Egresos de la Federación 200o-20o8. Secretaria de Hacienda y Crédito Público. Disponible en: http://www.apartados. hacienda.gob.mx/presupuesto/index.html.

UNIÓN, C. D. (2009). Constitución Política de los Estados Unidos Mexicanos. Mexico: UNIÓN.

Vargas, A. (1999). Notas sobre el Estado y las Políticas Públicas. Bogotá D.C.: Almudena Editores.

Zermeño, J. (2009, febrero 15). Optan Estados por mando militar. Reforma, suplemento Enfoque. 
